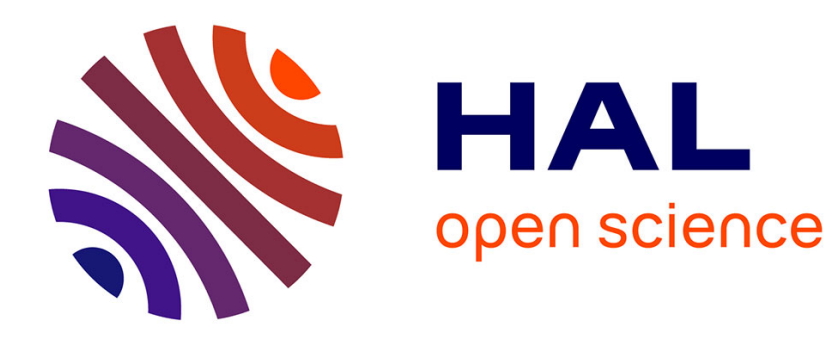

\title{
Les inégalités écologiques et sociales : l'apport des théories de la justice
}

Fabrice Flipo

\section{To cite this version:}

Fabrice Flipo. Les inégalités écologiques et sociales : l'apport des théories de la justice. Mouvements: des idées et des luttes, 2009, 60, pp.59-76. 10.3917/mouv.060.0059 . hal-00957966

\section{HAL Id: hal-00957966 https://hal.science/hal-00957966}

Submitted on 11 Mar 2014

HAL is a multi-disciplinary open access archive for the deposit and dissemination of scientific research documents, whether they are published or not. The documents may come from teaching and research institutions in France or abroad, or from public or private research centers.
L'archive ouverte pluridisciplinaire HAL, est destinée au dépôt et à la diffusion de documents scientifiques de niveau recherche, publiés ou non, émanant des établissements d'enseignement et de recherche français ou étrangers, des laboratoires publics ou privés. 


\title{
Les inégalités écologiques et sociales : l'apport des théories de la justice
}

\author{
Fabrice Flipo \\ Maître de conférences en Philosophie \\ Telecom \& Management SudParis, laboratoire Cemantic \\ Fabrice.flipo@it-sudparis.eu
}

Parler d'inégalités revient implicitement à poser la question des origines des inégalités. La philosophie morale et politique est riche de théories expliquant l'origine des inégalités, soit pour les dénoncer, soit pour les justifier. A l'époque moderne, les inégalités sont abordées comme étant moins issues de la substance que des procédures - autrement dit, si vous êtes pauvre et peu intelligent ce n'est pas parce que vous êtes la réincarnation d'un voleur, comme le dirait le système des castes, mais parce que les règles du jeu social vous ont assigné cette place, à tort ou à raison.

La perspective d'une émancipation implique à la fois une meilleure sinon une égale répartition des biens (intelligence, beauté, richesse...) mais aussi des maux. Le défi posé par l'articulation des inégalités écologiques et des inégalités sociales est complexe en ce que la discussion porte tant sur la définition de ce qui est «bien» et de ce qui est «mal» (une voiture est-elle un bien ? un vélo ?) - ce qui se traduit notamment par l'émergence de "nouveaux indicateurs de richesse »- que sur leur répartition. Fabrice Flipo tente, dans un texte un peu dense, de faire un état des lieux.

\section{Résumé}

Savoir si une inégalité est légitime ou illégitime est l'un des objets des théories de la justice. Après avoir rappelé l'origine des notions «d'écologie » (ou « environnement ») et « social», et le flou qui les entoure, cet article procède en deux temps. Dans un premier temps, il s'intéresse aux manières dont les théories de la justice abordent les inégalités écologiques ou environnementales. Dans un second temps, il montre les tensions qui existent entre ces inégalités et les inégalités sociales.

\section{Summary}

Deciding whether an inequality is legitimate or not is a concern of theories of justice. After recalling the origins of the notions of "ecology" (or"environment") and "social", and the imprecision which surrounds them, this paper will first examine how theories of justice treat ecological and environmental inequalities and then will show the tensions which exist between these and social inequalities 
F. Flipo, Les inégalités écologiques et sociales: l'apport des théories de la justice, Mouvements, $n^{\circ} 60$, octobre-décembre 2009, pp. 59-76.

\section{Mots clés}

Ecologie, social, théories de la justice, philosophie, inégalités.

Ecology, social, theories of justice, philosophy, inequalities.

\section{Introduction}

Amartya Sen a montré que toutes les théories de la justice reposent sur une forme ou une autre d'égalité considérée comme étant à atteindre ${ }^{1}$. Ces inégalités reposent finalement sur deux types de diversité ${ }^{2}$ : la diversité entre les êtres humains et leur situation, et la diversité des variables grâce auxquels l'inégalité peut être jugée. Aucune théorie de la justice n'a jamais voulu imposer une égalité parfaite entre les êtres humains, toutes ont tenu compte d'inégalités légitimes. Ces inégalités sont l'expression de la diversité et le respect des individualités. La manière selon laquelle une société examine les inégalités est dépendant de ce qu'elle juge être légitime car établir une inégalité, c'est établir une discrimination. Classer les Français selon la couleur, par exemple, est considéré comme illégitime. "En droit, l'interdiction de principe de la discrimination tient au fait que celle-ci conduit à associer un critère de distinction considéré comme illégitime et un traitement plus favorable fondé sur ce critère $»^{3}$. De l'établissement des inégalités au «traitement plus favorable », il n'y qu'un pas. L'injustice ne réside donc pas dans la discrimination, mais dans la discrimination illégitime, c'est-à-dire injuste. Pour que les personnes soient traitées de manière juste, il est nécessaire de reconnaître les inégalités légitimes des inégalités illégitimes. Les travaux en matière d'inégalités permettent de nourrir ces débats.

Pour contribuer à éclairer cette problématique, cet article propose de revenir sur les théories de la justice qui fondent ces inégalités, et voir comment travaillent quelques-uns de leurs arguments.

L'appel à contribution évoque les inégalités « sociales » et « écologiques ». Toutefois, comme nous l'avons montré antérieurement dans Développement Durable et Territoires, « social » est un terme problématique ${ }^{4}$. Nous avions rappelé que depuis le $19^{\text {ème }}$ siècle, au moins, «l'économie » et « le social » ont émergé comme deux secteurs bien identifiés de l'activité humaine ou au moins occidentale. L'économie désigne le secteur de la production de « richesses » et le social est l'ensemble des activités permises par la redistribution de la richesse créée par ce premier secteur ${ }^{5}$ : est « socialisé » le revenu qui est mis en commun et gouverné par l'ensemble des citoyens assemblés, à la différence du revenu "privé ». L'économie renvoie à la gestion, la comptabilité etc. tandis que le social renvoie à l'emploi, aux syndicats, à la division du travail et aux droits de l'homme de deuxième génération. Social et économie sont interdépendants d'une même vision du monde.

L'appel ne fait guère de différence entre enjeux "écologiques » et enjeux « environnementaux ». Là aussi il faut rappeler que « l'environnement » émerge au milieu des années 60. Le Dictionnaire d'épistémologie et de philosophie des sciences indique que l'usage

\footnotetext{
${ }^{1}$ Sen A., Inequalities re-examined, Oxford, Clarendon Press, 1992.

${ }^{2}$ Sen A., ibid., p.1.

${ }^{3}$ Haut Conseil à l'Intégration, Lutte contre les discriminations : faire respecter le principe d'égalité. Rapport au Premier Ministre, Paris, La Documentation Française, 1998, p. 9.

${ }^{4}$ Flipo F., Les tensions constitutives du « développement durable, Développement Durable et Territoires, avril 2004. http://developpementdurable.revues.org/index1041.html

${ }^{5}$ Miller D., Coleman J., Connolly W. \& A. Ryan (ed.), Social-démocratie, pp. 713-719, Dictionnaire de la pensée politique - Hommes et idées, Paris, Hatier, Ed. orig. London, Basil Blackwell, 1989, pp. 713719.
} 
F. Flipo, Les inégalités écologiques et sociales: l'apport des théories de la justice, Mouvements, $n^{\circ} 60$, octobre-décembre 2009, pp. 59-76.

actuel est né dans les années $60^{6}$. De fait, le Dictionnaire étymologique de 1938 n'a pas d'entrée « environnement », nous n'y trouvons que "environ » avec la définition suivante : composé de l'ancien français "viron", dérivé de "virer", ronde, pays d'alentour". Rachel Carson, que l'on crédite souvent d'avoir inauguré cette crise, ne parle pas d'

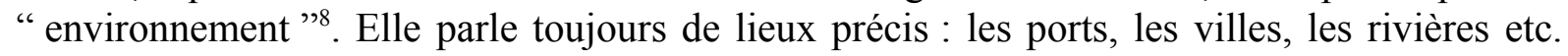
Dérivé de l'anglais, “c'est à la charnière des années 1960 et 1970 que le mot s'impose en France, dans son sens actuel, pour qualifier un domaine de responsabilité politique, plus que pour désigner un champ de recherche scientifique" . En première approximation, on peut dire que "la question de base suscitée par le champ de l'environnement est de savoir comment penser l'intégration de sujets humains et d'êtres non-humains dans une même représentation $\gg{ }^{10}$. L'environnement $\mathrm{n}$ 'est $\mathrm{ni}$ un intérêt catégoriel ni un problème de redistribution. Ce n'est pas une institution. Ce n'est pas non plus un facteur de production. Les problèmes d'environnement ne peuvent pas être vus, compris ni pris en charge par les sciences, institutions ou organismes existants mais par des entités interdisciplinaires et intersectoriels. «L'environnement », c'est plutôt le lieu des «effets pervers $»^{11}$, « accidents » et autres événements néfastes et imprévus produits par les deux autres catégories.

L'écologie elle-même a un périmètre mouvant. D'abord réservée à l'étude des écosystèmes séparés de toute activité humaine ${ }^{12}$, elle s'intéresse de plus en plus aux interactions entre nature et sociétés humaines. Le terme " écologie humaine » est né dans la sociologie urbaine des années vingt (Ecole de Chicago), non pas pour étudier la ville comme élément des écosystèmes mais pour utiliser l'écologie des plantes comme schème analogique pour analyser certains phénomènes urbains. L'analogie écologique a aussi été appliquée dans le domaine des idées, par exemple par Gregory Bateson ${ }^{13}$. Avant l'écologie humaine, l'historiographie n'a relevé que quelques précurseurs comme la géographe Elisée Reclus ou l'urbaniste Patrick Geddes ${ }^{14}$. Puis les biologistes ont repris le terme " écologie humaine» pour «montrer que les êtres humains sont sujets aux mêmes limitations écologiques que les autres animaux $»^{15}$. La Société Internationale d'Ecologie Humaine a été fondée en 1978 à l'initiative d'un médecin biologiste de Bordeaux. L'actuelle société d'écologie humaine (SEH) a été fondée en 1987 et définit l'écologie humaine comme « un questionnement où la relation entre l'humanité et la nature est abordée essentiellement à partir de la relation entre les populations humaines et leur environnement » (www.ecologie-humaine.eu). L'être humain est alors assimilé à un écosystème parmi d'autres, avec ses spécificités.

\section{Les approches classiques de la « justice écologique »}

\footnotetext{
${ }^{6}$ Larrère C. \& L., Environnement, in D. Lecourt (dir.), Dictionnaire d'histoire et de philosophie des sciences, Paris, PUF, 1999, pp. 360-363.

${ }^{7}$ Dauzat A., Dictionnaire étymologique de la Langue Française, Paris, Larousse, 1938, p283.

${ }^{8}$ Carson R., 1962, Silent Spring, Boston, Houghton Mifflin.

${ }^{9}$ Larrère C. \& L., op. cit., 1999.

${ }^{10}$ Godard O., Le concept d'environnement, une hiérarchie enchevêtrée, in Larrère C. \& R., La crise environnementale, Paris, INRA Editions, 1994, pp. 97-112.

${ }^{11}$ Jonas H., Le principe responsabilité, Paris, Flammarion, 1990, Ed. orig. 1979, p. 350.

${ }^{12}$ Deléage J.-P. , Histoire de l'écologie, Paris, La Découverte, 1992.

${ }^{13}$ Bateson G., Vers une écologie de l'esprit, Paris, Seuil, 1995.

${ }^{14}$ Deléage J.-P. , op. cit. , p. 68.

${ }^{15}$ Marten G.G., Human ecology - basic concept for sustainable development, London, Earthscan, 2001, p. XV.
} 
F. Flipo, Les inégalités écologiques et sociales: l'apport des théories de la justice, Mouvements, $n^{\circ} 60$, octobre-décembre 2009, pp. 59-76.

Que sont les théories «écologiques » de la justice ? Les théories de la justice ne parlent que de justice, rarement de "justice écologique »: l'article «Justice» du dictionnaire de philosophie éthique et morale ne l'évoque même pas ${ }^{16}$.

Le flou qui entoure le terme « écologie » explique une bonne partie de la difficulté qu'il y a à cerner des inégalités qui puissent mériter le qualificatif d' « écologique ». Ici nous retiendrons comme « écologiques » les théories de la justice qui se sont explicitement réclamées en faveur « de l'écologie », autrement dit les théories écologistes, ou environnementalistes.

Nous aborderons ici les différentes théories de la « justice écologique » en les regroupant sous trois formes principales : l'éthique environnementale, l'utilitarisme et l'approche patrimoniale (droits de propriété, propriété privée et biens communs). Cette typologie est une proposition abordant un champ mal identifié dont nous tentons de démontrer la cohérence au fil de l'argumentation.

L'éthique environnementale est une approche centrée sur le rapport de l'individu à la nature, celle-ci étant généralement appréhendée par l'écologie en tant que science de la nature. Cette approche exclut donc largement les activités humaines ${ }^{17}$. L'écologie est aujourd'hui scindée en différentes écoles dont "l'unité de base» est différente ${ }^{18}$ : écosystèmes, populations, organismes, cycles biogéochimiques etc. Ces unités de base sont imbriquées les unes dans les autres et donnent naissance à des "systèmes" dont la logique d'évolution varie selon l'échelle à laquelle ils sont observés.

Dans cette masse de causes et de conséquences imbriquées, les éthiques environnementales tentent de discerner les moyens des fins ${ }^{19}$. Les écosystèmes doivent-ils être considérés comme des moyens en vue de servir la vie des organismes ou au contraire les écosystèmes doivent-ils être considérés comme des fins en soi, pour assurer la pérennité de l'espèce ? Quelle est la hiérarchie entre les espèces ? Jusqu'à où l'être humain peut-il intervenir ? A partir de quel moment doit-il considérer qu'il outrepasse ses droits et laisser faire la nature ? Ce sont des approches généralement déontologiques c'est-à-dire qu'elles cherchent à « les actes qui sont moralement obligatoires ou prohibés, sans égards pour leurs conséquences dans le monde $»^{20}$. Elles peuvent toutefois faire appel à des approches plus conséquentialistes, qui «évaluent un choix en examinant ses conséquences »"

Les éthiques environnementales se scindent en différents courants. La position la plus restrictive est le biocentrisme. Pour P. Taylor, la maxime de l'action juste est de traiter à égalité tous les vivants ${ }^{22}$, êtres humains ou non. Le respect est déterminé en fonction des intérêts que l'être vivant en question cherche à poursuivre, que l'on considère ici comme étant légitimes et dont la continuité doit être assurée. L'action humaine doit respecter ces finalités, et indirectement leurs conditions de possibilité c'est-à-dire l'intégrité des habitats. On peut

\footnotetext{
${ }^{16}$ Audard C., Justice, in M. Canto-Sperber (dir.), Dictionnaire d'Ethique et de Philosophie Morale, Paris, 1996, PUF, pp. 781-789.

${ }^{17}$ Larrère C., Les philosophies de l'environnement, Paris, PUF, 1997a, p. 6.

${ }^{18}$ Ramade F., Eléments d'écologie - 2 tomes, Paris, Ediscience international, 2003.

${ }^{19}$ Larrère C., op. cit., 1997a, p. 18.

${ }^{20}$ Berten A., Déontologisme, in M. Canto-Sperber (dir.), Dictionnaire d'Ethique et de Philosophie Morale, Paris, PUF, 1996, p. 378.

${ }^{21}$ Pettit P., Conséquentialisme, in M. Canto-Sperber (dir.), Dictionnaire d'Ethique et de Philosophie Morale, Paris, PUF, 1996, p. 313.

${ }^{22}$ Taylor Paul W., In Defense of Biocentrism, Environmental Ethics, Vol. 5, No. 3 (Automne), 1983, pp. 237-243.
} 
F. Flipo, Les inégalités écologiques et sociales: l'apport des théories de la justice, Mouvements, $n^{\circ} 60$, octobre-décembre 2009, pp. 59-76.

donc modifier les écosystèmes à volonté, pourvu que les vivants, y compris les humains, en bénéficient.

Les courants de l'éthique animale sont proches du biocentrisme, mais limitent leur analyse à la prise des animaux en tant qu'êtres vivants capables de souffrance ${ }^{23}$. Ils affirment par exemple que l'on peut tuer des animaux pourvu qu'ils ne souffrent pas et que cela soit fait pour des raisons valables (se nourrir par exemple). Indirectement, on peut en déduire un devoir de protection des écosystèmes, de manière à ce que les modifications d'origine humaine ne provoquent pas de souffrance chez les animaux. Elle peut aussi parfois préconiser de soigner un animal blessé par un homme ${ }^{24}$. Par exemple c'est le cas avec les animaux domestiques ou avec les oiseaux sauvages secourus lors du naufrage de l'Erika.

L'écocentrisme de J.B. Callicott affirme par contre qu'une « action est juste quand elle ne perturbe la communauté biotique qu'à une échelle spatiale et temporelle normale $»^{25}$. Ici, on peut tuer quelques animaux pourvu que l'équilibre général de la communauté biotique, c'est-àdire son intégrité, soit maintenu. Les écosystèmes sont envisagés à égalité avec les intérêts humains. Tout le problème est de définir ce qu'on entend par « normal », concept qui doit être pris en son sens axiologique. Dans une relecture de Callicott à l'aune des connaissances les plus récentes en matière d'écologie, C. \& R. Larrère proposent la diversité biologique comme critère d'évaluation ${ }^{26}$. La richesse biologique est en effet aujourd'hui reconnue comme un bon moyen pour accroître la résilience d'un écosystème. Elle comprend la diversité domestique et la diversité sauvage.

La seconde approche peut être dite «utilitariste», qui recouvre au sens classique trois critères: "une action ne peut être jugée bonne ou mauvaise qu'en raison de ses conséquences sur le bonheur des individus concernés ", l'utilité doit être maximisée sous différents critères qui sont tous conséquentialistes ${ }^{27}$. Dominée par l'économie, dont Amartya Sen rappelle qu'elle est une science morale ${ }^{28}$, c'est une approche souvent " anthropocentrée » c'est-à-dire qu'elle évalue les coûts et bénéfices uniquement pour l'être humain. Certaines théories étendent cependant cette comptabilité à tous les êtres sensibles ${ }^{29}$. Dans le domaine des inégalités intergénérationnelles, l'utilitarisme écologique se scinde en plusieurs courants, qui convergent tous vers ce qu'A. Gosseries appelle « l'interdiction de désépargne » c'est-àdire que chaque génération a pour consigne d'effacer toute conséquence négative de ses $\operatorname{actes}^{30}$.

Les partisans de la soutenabilité dite « faible » s'appuient sur la théorie économique standard et affirment qu'il n'existe aucune ressource naturelle à protéger car la technologie permet de continuer à augmenter le revenu disponible ${ }^{31}$. Quand une ressource se fait plus rare, le prix augmente et les moyens sont dégagés pour trouver d'autres ressources, maintenant ainsi le

\footnotetext{
${ }^{23}$ Singer P., La libération animale, Paris, Grasset, 1993 ; Bentham J., Introduction to the Principles of Morals and Legislation, 1780, chap. 17.

${ }^{24}$ Fritsch P., Effets croisés de réflexivité : prises de position problématiques sur la sauvegarde de la faune sauvage, Aubert F. \& J.-P. Sylvestre (dir.), Ecologie et société, Paris, Educagri Editions, 1998, pp. 81-93.

${ }^{25}$ Larrère C., op. cit., $1997 a$, p. 84.

${ }^{26}$ Larrère C. \& R., op. cit., 1997b, p. 281.

${ }^{27}$ Audard C., Utilitarisme, in, M. Canto-Sperber (dir.), Dictionnaire d'Ethique et de Philosophie Morale, Paris, PUF, 1996, p. 1563.

${ }^{28}$ Sen A., L'économie est une science morale, Paris, La Découverte, 2004.

${ }^{29}$ Singer P., op. cit.; Bentham J., op. cit.

${ }^{30}$ Gosseries A., De l'affaire Perruche a la réforme des retraites, Paris, Aubier-Flammarion, 2004.

${ }^{31}$ Solow R. M., The Economics of Resources or the Resources of Economics, pp. 1-14, The American Economic Review, vol. 64, n², Nashville, May 1974, p. 10.
} 
F. Flipo, Les inégalités écologiques et sociales: l'apport des théories de la justice, Mouvements, $n^{\circ} 60$, octobre-décembre 2009, pp. 59-76.

niveau global d'utilité. Les ressources sont substituables entre elles : quand le pétrole s'épuise, le charbon prend le relais, et ainsi de suite dans tous les domaines. Solow et d'autres appuient leurs travaux sur des mesures faites aux Etats-Unis entre 1890 et $1960^{32}$. Puisque les générations à venir seront plus riches, elles auront davantage de moyens pour lutter contre les pollutions. R. Solow estime que nous ne serions amenés à ne conserver que les sites remarquables, sur le plan esthétique, tels que le Yosemite Park. Pour cette approche, vouloir conserver le pétrole ou les écosystèmes est absurde : nous devons au contraire les consommer car ce sont les bénéfices que la société peut en tirer qui comptent. Cet argument est renforcé par la thèse des "courbes de Kuznets $»^{33}$ qui montrent qu'avec le début du développement économique la pollution augmente puis, au-delà d'un point de retournement, elle diminue car la société s'équipe de technologies propres. Le libre-échange apparaît dès lors comme la solution puisqu'il permet la diffusion de technologies propres.

Les partisans de la durabilité « forte » affirment au contraire que certaines ressources ne sont pas substituables et doivent être protégées comme des fins en soi : elles ont une valeur intrinsèque pour le bonheur humain, elles sont utiles en elles-mêmes, et leur transformation constitue une perte difficilement compensable. Ce statut est tiré des limites de la technique : certaines dégradations sont irréversibles, la "courbe de Kuznets » n'existe pas pour les déchets et la plupart des pollutions globales, l'empreinte écologique des pays industrialisés ne cesse d'augmenter, les effets de dématérialisation étant en partie provoqués par la délocalisation des activités les plus polluantes ${ }^{34}$. La substitution des ressources revient en réalité à les épuiser les unes après les autres, laissant finalement le monde dans un état de haute entropie. Les partisans de la durabilité forte pensent davantage en termes de reproduction de l'économie qu'en termes de croissance et ils affirment que l'économie doit s'adapter à l'écologie et non l'inverse. L'un des précurseurs français en la matière est René Passet, avec son ouvrage L'économique et le vivant paru en $1979^{35}$. Dans le monde anglosaxon la référence est Hermann Daly, le fondateur de la revue Ecological Economics ${ }^{36}$. De ce point de vue, les revenus croissants sont le fruit d'un endettement croissant, contrevenant à l'interdiction de désépargne. Tout l'enjeu est alors de mettre en place un système économique capable de créer une richesse pérenne, qui soit renouvelable en droit. Pour beaucoup d'entre eux, le seul critère monétaire ne saurait suffire : les analyses utilisent volontiers des matrices multicritères. R.U. Ayres par exemple établit la liste suivante de critères de durabilité forte ${ }^{37}$ : pas de changement supplémentaire dans le climat, stabilisation de l'acidité des pluies, pas d'accumulation supplémentaire de toxiques, fin à tous les usages dissipatifs des métaux rares et des ressources provenant de l'extraction, pas de pompage supplémentaire de l'eau fossile, pas de perte supplémentaire de sol et pas de perte supplémentaire de milieux (zones humides etc.)... En pratique l'interprétation de ces critères est très large et varie beaucoup selon les écoles, qui vont de la durabilité très forte à la durabilité assez faible... Suivant en cela les travaux de Nicholas Georgescu-Roegen ${ }^{38}$, certains partisans de la durabilité forte vont jusqu'à

\footnotetext{
${ }^{32}$ Vivien F.-D., Le développement soutenable, Paris, La Découverte, 2005.

${ }^{33}$ Zuindeau B., La «loi de Kuznets » : de l'économie de la répartition à l'économie de l'environnement, Colloque international Association Charles Gide pour l'Etude de la Pensée Economique, 2005.

${ }^{34}$ Bringzu S. \& H. Schütz, Total material requirements of the European Union, Technical Report no 55, European Environment Agency, 2001, p.5.

${ }^{35}$ Passet R., L'économique et le vivant, Paris, Economica, 1979.

${ }^{36}$ Daly H.E. \& J. Cobb Jr., For the Common Good, Boston, Beacon Press, 1989.

${ }^{37}$ Ayres R.U. \& L.W., Accounting for resources 2 - The life cycle of materials, Edward Elgar

Publishing, 1999, p. 257.

${ }^{38}$ Georgescu-Roegen N., La décroissance, Paris, Editions Sang de la Terre, 1979.
} 
F. Flipo, Les inégalités écologiques et sociales: l'apport des théories de la justice, Mouvements, $n^{\circ} 60$, octobre-décembre 2009, pp. 59-76.

affirmer que la décroissance des revenus est une conséquence logique de la prise en compte de la richesse écologique ${ }^{39}$, ce que d'autres récusent.

Dans le domaine des inégalités intragénérationnelles, les travaux sont plutôt microéconomiques ou sectoriels. Ils portent d'un côté sur les nuisances (bruit, proximité avec les décharges, exposition aux toxiques tels que les polluants organiques persistants $)^{40}$ et de l'autre sur l'internalisation des externalités (écofiscalité, pollueur-payeur). Toutes ces problématiques sont anciennes mais elles n'ont eu qu'un écho limité sur les politiques publiques jusqu'ici. Les « maladies environnementales »n'ont commencé à réellement émerger qu'avec l'Appel de Paris lancé par l'Artac, sous la houlette du professeur Belpomme ${ }^{41}$. Elles rejoignent les thèses d'U. Beck sur la société du risque : alors que pendant longtemps les sociétés industrielles ont développé des systèmes de réduction des risques, aujourd'hui elles sont à l'origine de nouveaux risques ${ }^{42}$. Désormais la division du travail ne crée plus seulement des gains de productivité mais aussi des gains de «destructivité». L'étude européenne portant sur le principe de précaution montre les difficultés rencontrées en matière d'étude des conséquences négatives de l'industrie sur l'environnement ${ }^{43}$. En explorant l'histoire, les théoriciens de la justice écologique se trouvent des précurseurs dont les thèses ont été largement ignorées de leur vivant. Une partie de ces théories sont dans la zone grise entre « santé », "ressources » etc. et n'entrent pas forcément dans «l'écologie » proprement dite, du nous retrouvons ici la difficulté à classer évoquée plus haut. La thématique de l'internalisation des externalités peut aussi être considérée comme appartenant à l'utilitarisme car il s'agit ici de corriger des imperfections de marché en faisant en sorte que les prix de marché reflètent les utilités réelles. Elle couvre un vaste domaine qui va de méthodes très microéconomiques comme l'évaluation contingente jusqu'à des analyses macroéconomiques ou macroécologiques telles que l'évaluation monétaire des services rendus par la biosphère ${ }^{44}$. Elle intègre aussi l'ensemble des théories qui portent sur les mécanismes correcteurs: écotaxes et réglementation administrative de diverses émissions.

J. Martinez-Alier fait le bilan des analyses écologiques dans le domaine des inégalités internationales ${ }^{45}$ et montre que les approches classiques ont ignoré ce facteur jusqu'à la fin des années $80^{46}$. Martinez-Alier définit l'échange écologiquement inégal comme «le fait d'exporter des produits des régions et pays pauvres à des prix qui ne tiennent pas compte des externalités locales causées par ces exportations ou par l'épuisement des ressources naturelles en échange de biens et de services en provenance des pays riches ${ }^{47}$. A la classique dégradation des termes de l'échange sont ajoutées les externalités, payées au prix « local » c'est-à-dire sous-évaluées, et l'épuisement des richesses naturelles. L'auteur a donné

\footnotetext{
${ }^{39}$ Cochet Y., Pétrole apocalypse, Paris, Fayard, 2006.

${ }^{40}$ Theys J., Les inégalités écologiques, dimension oubliée de l'action publique : entre raisons politiques et explications épistémologiques, Colloque « Inégalités sociales et environnementales », Institut d'Urbanisme de Paris, 2005.

${ }^{41}$ Belpomme D., Ces maladies créées par l'homme, Paris, Albin Michel, 2004.

${ }^{42}$ Beck U., La société du risque - sur la voie d'une autre modernité, Paris, Flammarion, 2003.

${ }^{43}$ IFEN, Signaux précoces et leçons tardives : le principe de précaution 1896-2000, Agence

Européenne de l'Environnement, 2001.

${ }^{44}$ Costanza R. \& al., The value of the world's ecosystem services and natural capital, Nature, vol. 387, 1997, pp. 253-260.

${ }^{45}$ Cabeza-Gutés M. \& J. Martinez-Alier, L’échange écologiquement inégal, M. Damian \& J.-C. Graz, Commerce international et développement soutenable, Paris, Economica, 2001 ; Muradian R. \& J. Martinez-Alier, Trade and the environment : from a « Southern » perspective, Ecological Economics, 2001, n³6, pp. 281-297.

${ }^{46}$ Cabeza-Gutés M. \& J. Martinez-Alier, op. cit., p171

${ }^{47}$ Cabeza-Gutés M. \& J. Martinez-Alier, op. cit., p172
} 
F. Flipo, Les inégalités écologiques et sociales: l'apport des théories de la justice, Mouvements, $n^{\circ} 60$, octobre-décembre 2009, pp. 59-76.

$\mathrm{du}$ contenu au concept de $"$ dette écologique $»^{48}$, issu des mouvements associatifs. A. Hornborg développe de son côté la thèse selon laquelle l'échange est un jeu à somme nulle, du point de vue écologique : les biens et les maux écologiques sont simplement déplacés, additionnés ou soustraits ${ }^{49}$. Le Wuppertal Institute et le SERI ont développé des comptabilités matérielles qui montrent que la dématérialisation apparente des économies les plus riches cache une augmentation du «sac à dos écologique», comme évoqué plus haut. Plus généralement, l'ensemble des analyses liées au courant de "l'économie écologique » (ecological economics) affirme que la question d'une économie écologique est principalement un problème de taille de l'économie ${ }^{50}$, et non de composition ou de répartition. S. Guha \& J. Martinez-Alier affirment même que "la richesse est un plus grand danger pour l'environnement que la pauvreté ${ }^{51}$. Les récents développements autour de l'empreinte écologique vont aussi dans ce sens ${ }^{52}$.

Articuler les inégalités nationales et les inégalités internationales est l'un des plus gros problèmes actuels. Un smicard consomme au moins l'équivalent d'une planète et demi, si l'on suit les évaluations de J.-M. Jancovici" ${ }^{53}$, c'est donc un "riche » écologique du point de vue planétaire, quoique « pauvre » du point de vue national. La tonne de carbone a-t-elle la même valeur au Bangladesh qu'en France? La voiture, nécessaire dans une ville comme Houston, n'est-elle pas superflue dans bon nombre de villages africains? Dès lors qui est riche, l'Américain obligé de prendre sa voiture ou l'Africain capable de s'en passer? Les comparaisons sont très difficiles à faire car les contextes sont très différents. Les macrosystèmes techniques voraces mis en place par les pays industrialisés n'apportent plus beaucoup d'améliorations de la qualité de vie, comme le montrent les indicateurs alternatifs tels que le Genuine Progress Indicator (www.rprogress.org) ou la carte mondiale du bonheur produite par l'Université de Leicester (trouvable sur internet). C'est pourtant dans ce domaine que les enjeux sont les plus élevés, car ce sont eux qui font le plus débat dans l'espace international et selon toute vraisemblance ce sont eux qui ont le potentiel de conflit le plus grand. Les comparaisons sont complexes et fortement multicritères, comme en témoigne la discussion de l'équité dans la négociation sur les changements climatiques ${ }^{54}$.

La dernière approche est celle des droits, et en son sein des droits de propriété. Elle se scinde à son tour en différentes approches. Sa caractéristique est d'aborder les questions en termes de souveraineté, individuelle ou collective, et non d'éthique ou de quantité de bonheur ou d'utilité.

\footnotetext{
${ }^{48}$ Martinez-Alier J., Ecological Debt - External Debt, 1998, http://www.cosmovisiones.com/DeudaEcologica/a_alier01in.html; Paredis E. \& al., Elaboration of the concept of ecological debt, VLIR-BVO project 2003, Final report. , 2004 http://cdonet.rug.ac.be/noordzuid/onderzoek/ecological_debt/ecodebt_report_1_core.pdf

${ }^{49}$ Hornborg A., Cornucopia or zero-sum game? The epistemology of sustainability, Journal of WorldSystems, vol. 9, n², 2003, pp. 205-216; Hornborg A., Footprints in the cotton fields: The Industrial Revolution as time-space appropriation and environmental load displacement, Ecological Economics, n59, 2006, pp. 74-81.

${ }^{50}$ Daly H., Beyond Growth, Boston, Beacon Press, 1996, pp. 59-60.

${ }^{51}$ Guha R. \& J. Martinez-Alier, Varieties of environmentalism, London, Earthscan, 2000, Ed. Orig. 1997, p. 59.

${ }_{52}^{52}$ WWF, Living Planet Report, 2006 ; Wackernagel M. \& W. Rees, Notre empreinte écologique, Montréal, Ecosociété, 1996.

${ }^{53}$ Jancovici J.-M., Combien suis-je un esclavagiste ? , 2005. http://www.manicore.com/documentation/esclaves.html

${ }^{54}$ Toth F. L. (Ed.), Fair Weather? Equity concerns in Climate Change, Earthscan, London, 1999 ; Flipo F., La question de l'équité dans le cas du changement climatique, Ethique et économique/Ethics and Economics, 2 (1), 2004, http://Ethique-economique.org
} 
F. Flipo, Les inégalités écologiques et sociales: l'apport des théories de la justice, Mouvements, $n^{\circ} 60$, octobre-décembre 2009, pp. 59-76.

La plupart des théories partent des approches gestionnaires des communaux tels que les eaux et forêts. Elles sont souvent tirées de l'observation des réglementations de l'Etat. F. Ost ${ }^{55}$ et d'autres ${ }^{56}$ ont ainsi montré que les biens communs sont instituables sous la forme de patrimoine commun. De fait la réglementation en vigueur est plutôt de type patrimonial, que ce soit en France ou à l'échelle mondiale. Les critères de R.U. Ayres cités plus haut sont finalement eux aussi de type patrimonial. D. Birnbacher, parti pour établir une analyse utilitariste de la justice intergénérationnelle, finit lui aussi par retomber sur des critères de ce genre $^{57}$. C. Larrère note que les approches coûts / bénéfices conduisent très souvent à des calculs complexes et insolubles ${ }^{58}$. Le patrimoine articule des droits et des devoirs individuels de telle manière à assurer la continuité d'un effet collectif global positif. Les économistes du PNUD ont réactivé la notion de « bien public ${ }^{59}$. Leur analyse des biens publics mondiaux a ouvert à de nouveaux travaux à d'autres échelles de gouvernance. Ici un régulateur central vient articuler intérêts particuliers et intérêt général pour réduire les inégalités intragénérationnelles et les inégalités intergénérationnelles.

J. Martinez-Alier a forgé la thèse d'un « environnementalisme des pauvres » qui prendrait volontiers la forme de du «narodnisme », encore nommé « agrarianisme ». Cette approche est basée sur une défense du mode de vie et des ressources propres, avec comme héros le mouvement Chipko (Inde) ou la lutte de Chico Mendes en Amazonie ${ }^{60}$. Ces mouvements s'appuient bien sur les droits : plutôt que de discuter des conséquences de telle ou telle action sur le bonheur collectif, c'est la question de la souveraineté qui est mise en jeu. C'est aussi l'approche de mouvements tels que Via Campesina qui défendent la souveraineté alimentaire ou du travail de L. Zarsky sur les droits de l'homme et l'environnement, qui se concentre sur le droit des communautés ${ }^{61}$. Le mouvement " environmental justice » peut aussi figurer dans la catégorie de la justice écologique basée sur les droits. M. William Clinton a promulgué le 11 février 1994 l'Executive Order 12898 par lequel il demande aux agences fédérales, et en particulier à l'EPA (Environmental Protection Agency - l'agence fédérale de protection de l'environnement), de promouvoir une politique visant à éviter toute discrimination de race, sexe, religion, niveau de revenu dans la distribution des risques environnementaux. Ces mouvements sont encore faibles et plutôt soucieux de risques locaux ${ }^{62}$. Toutefois ces mouvements se revendiquent rarement comme « écologiques » et ne sont pas toujours reliés à un souci de justice au sens global du terme. Un auteur comme A. Dobson doute même de ce qu'ils soient réellement en faveur de la soutenabilité écologique ${ }^{63}$. L'ambiguïté de ces mouvements vient plutôt de ce que le terme "environnemental » ou " écologique " parait surimposé ou capté de manière opportuniste par les mouvements eux-mêmes. Cela traduit-il quelque chose de nouveau ? Ou est-ce simplement une manière nouvelle de rebaptiser des

\footnotetext{
${ }^{55}$ Ost F., La nature hors la loi. L'écologie à l'épreuve du droit, Paris, La Découverte, 1995.

${ }^{56}$ Larrère C. \& R., op. cit., 1997b, p. 189.

${ }^{57}$ Birnbacher D., La responsabilité envers les générations futures, Paris, PUF, 1998, p. 188-225.

${ }^{58}$ Larrère C., op. cit., 1997a, p. 23.

${ }^{59}$ Kaul I., Grunberg I. \& A. Stern, Global Public Goods - International Cooperation in the $21^{\text {st }}$ Century, Oxford, Oxford University Press, 1999.

${ }^{60}$ Martinez-Alier J., The environmentalism of the poor, A report for UNRISD for the WSSD Summit, 2002. http://www.foe-scotland.org.uk/nation/ej_alier.pdf

${ }^{61}$ Zarsky L. (Ed.), Human rights and the environment - conflicts and norms in a globalizing world, London, Earthscan, 2002.

${ }^{62}$ Faber D.R. \& D.McCarthy,Neo-liberalism, globalization and the struggle for ecological democracy: linking sustainability and environmental justice, 2003, pp. 37-63; Agyeman J., Bullard R. D. \& B. Evans, Just sustainabilities - Development in an unequal world, London, Earthscan.

${ }^{63}$ Dobson A., op. cit., 2003, pp. 83-95; Agyeman J., Bullard R. D. \& B. Evans, op. cit., p. 85.
} 
F. Flipo, Les inégalités écologiques et sociales: l'apport des théories de la justice, Mouvements, $n^{\circ} 60$, octobre-décembre 2009, pp. 59-76.

problèmes anciens, de manière à s'adapter aux représentations de l'oppresseur ? La réponse n'est pas aisée.

La question de la régulation du régulateur divise les théoriciens et renouvelle des questions classiques de philosophie morale et politique. Du côté libertarien, la thèse principale est posée par l'article fameux de Garrett Hardin : les biens communs sont voués à l'épuisement tant que la propriété privée n'est pas instaurée et respectée ${ }^{64}$. La propriété privée responsabilise les personnes alors qu'une propriété commune c'est une propriété qui n'est à personne. Les inégalités de propriété sont le prix à payer pour garantir l'absence d'inégalités intergénérationnelles. D'autres comme Murray Bookchin ${ }^{65}$, Jean Zin ${ }^{66}$ ou Serge Latouche ${ }^{67}$ objectent que la propriété privée de type capitaliste est échangeable et son strict respect conduit à la destruction des communaux. Pour eux, le problème réside dans l'omniprésence de l'intérêt économique, destructeur des communaux. L'appropriation de la nature ne connaît plus de limites. Ils prônent un "réenchâssement» de l'économique dans l'écologique, une inversion du mouvement qui a eu lieu au $19^{\text {ème }}$ siècle et qu'a décrit K. Polanyi ${ }^{68}$ dans le domaine social. Ils estiment que la dissociation qui a lieu aujourd'hui est allée beaucoup trop loin. Pour eux la crispation contemporaine sur la propriété privée est un symptôme du mal qui détruit les biens communs et non une solution.

\section{Des tensions fortes avec la « justice sociale »}

Les théories de la justice ne guère plus de «social» que d'écologie. Appliquant la même règle que pour les théories « écologiques », nous retiendrons comme « sociales » les théories de la justice qui se sont explicitement réclamées en faveur « du social» : ce sont les théories socialistes.

\section{Le social ignore encore largement l'écologie}

Les théories socialistes sont nombreuses. Elles sont pour une large part issues de l'œuvre de Marx. Quand ce dernier meurt, en 1883, le terme « écologie » est peu courant puisqu'Ernst Haeckel l'invente en $1866^{69}$ et Tansley définit «l'écosystème » en $1935^{70}$. Il est donc difficile de lui reprocher de n'avoir pas théorisé les inégalités "écologiques» ou leurs causes. Toutefois l'écologie ne réinvente pas entièrement ce qu'on appelait «l'histoire naturelle $»^{71}$ ou encore $"$ l'économie naturelle $\rangle^{72}$. Les « écosocialistes » actuels montrent que Marx avait bel et bien vu que la nature était l'une des sources de la richesse ${ }^{73}$. Marx accuse en effet le profit de spolier les ouvriers et la nature ${ }^{74}$. Ils soulignent toutefois ailleurs que Marx a négligé

\footnotetext{
${ }^{64}$ Hardin G.W., The Tragedy of the Commons, Science, $n^{\circ} 162,1968$, pp. 1243-1248.

${ }^{65}$ Bookchin M., Une société à refaire, Lyon, Atelier de création libertaire, 1992.

${ }^{66} \mathrm{http}: / /$ perso.orange.fr/marxiens

${ }^{67}$ Latouche S., Justice sans limites - le défi de l'éthique dans une économie mondialisée, Paris, Fayard, 2003.

${ }^{68}$ Polanyi K., La grande transformation - Aux origines politiques et économiques de notre temps, Paris, Gallimard, 1983, Ed. orig. 1944.

${ }^{69}$ Deléage J.-P. , op. cit., 1992, p. 61.

${ }^{70}$ Ibid., p. 102

${ }^{71}$ Larrère C. \& R., op. cit., 1997b, p. 75.

${ }^{72}$ Worster D., Les pionniers de l'écologie, Paris, Sang de la Terre, 1992, Ed. orig. 1985, p. 52.

${ }^{73}$ Iselin F., Ecologie et conscience de classe, pp. 33-43, M. Löwy (dir.), Ecologie et socialisme, Paris, Syllepse, 2005.

${ }^{74}$ Marx K., Le Capital, Paris, PUF, 1993, Ed. orig. 1872, p. 567.
} 
F. Flipo, Les inégalités écologiques et sociales: l'apport des théories de la justice, Mouvements, $n^{\circ} 60$, octobre-décembre 2009, pp. 59-76.

la controverse entre Engels et Podolinsky ${ }^{75}$. Les mouvements socialistes ne vont guère accorder d'importance aux enjeux écologiques. De fait, les théories « sociales » de la justice s'appuient sur une sociologie ancrées dans ce que R.E. Dunlap a appelé, dès 1978, le « human exemptionnalism paradigm ${ }^{76}{ }^{76}$ c'est-à-dire dans le présupposé que l'homme n'est pas concerné par les régulations écologiques qui régissent le comportement des autres espèces dans leur milieu. Sur le plan écologique tous les socialismes se sont comportés comme les capitalismes, difficile de laisser cet élément de côté. En pratique, à nouveau, les luttes écologistes se sont souvent opposées aux luttes ouvrières ${ }^{77}$.

Quand le marxisme s'est ouvert à l'écologie, très récemment, c'est principalement par la voie de la thèse d'une double exploitation, du prolétariat et de la nature ${ }^{78}$, conduisant à une "seconde contradiction" du capitalisme. Cette approche ne change quasiment rien à l'approche orthodoxe, la seconde contradiction poussant comme la première à la chute du capitalisme qui ferait advenir le communisme. Le travail dialectique des inégalités reste facteur de progrès et de libération des forces productives, l'écologie n'étant qu'une variable supplémentaire à internaliser.

Sans se réclamer de «l'écosocialisme », F. Chesnais et C. Serfati récusent cette thèse trop classique de la « seconde contradiction » en montrant que dans le cas de l'environnement «le capital représente une barrière, ou plus exactement une menace pressante pour l'humanité et dans l'immédiat pour certaines parties précises de celle-ci mais non pour le capital luimême $»^{79}$. Il se pose donc la question de savoir qui va défendre les régulations écosystémiques, si ce ne sont ni les capitalistes ni les ouvriers. J.-M. Harribey reconnaît d'ailleurs qu'une "difficulté majeure reste à résoudre pour l'avancée d'un paradigme écologique marxien : quelles forces sociales sont susceptibles de porter un projet majoritaire démocratique de transformation de la société pour aller dans le sens d'une meilleure justice vis-à-vis des classes les plus démunies et des générations à venir ? ${ }^{80}$.

Le marxisme n'est pas la seule pensée «sociale» à avoir négligé l'écologie. Du côté de l'éthique, Paul Ricœur, Emmanuel Lévinas et tant d'autres ont largement négligé les écosystèmes et les animaux. Il y a de très fortes raisons à cela. Pour les théoriciens " sociaux", admettre que l'homme soit un animal "comme les autres", pris dans les régulations écosystémiques et la chaîne alimentaire, c'est souvent remettre en cause l'humanisme lui-même. Alors que les théories écologiques tentent de discuter des liens entre êtres humains et nature, la justice sociale a généralement situé la racine des racismes voire des totalitarismes dans la «biologisation » de l'être humain, à la suite d'Hannah Arendt ${ }^{81}$ et de

\footnotetext{
${ }^{75}$ Guha R. \& J. Martinez-Alier, Varieties of environmentalism, London, Earthscan, 2000, Ed. Orig. 1997, p. 29.

${ }^{76}$ Dunlap R. E., The coming age of environmental sociology : an american perspective, pp. 25-40, Europaea, 1-2, anno VIII, 2002, p. 34.

${ }^{77}$ Vadrot P.-M., Déclaration des droits de la nature, Paris, Stock, 1973, p. 185 ; Sainteny G., L'introuvable ecologisme français, Paris, PUF, 2000, p. 153.

${ }^{78}$ O'Connor J., La seconde contradiction du capitalisme : causes et conséquences, pp. 57-66, Harribey J.-M. \& M. Loewy (dir.), Capital contre nature, Paris, PUF, 2003.

${ }^{79}$ Chesnais F. \& C. Serfati, L'origine et la nature des menaces pesant sur la reproduction de la vie de classes et de pays donnés : quelques outils analytiques et fils conducteurs marxistes, 2004. http://www.europe-solidaire.org/spip.php?article891.

${ }^{80}$ Harribey J.-M., Marxisme écologique ou écologie politique marxienne, pp. 183-200, Bidet J., Kouvélakis E. (dir.), Dictionnaire Marx contemporain, Paris, PUF, Actuel Marx Confrontation, 2001, p. 200.

${ }^{81}$ Arendt A., Sur l'antisémitisme, Paris, Calmann-Lévy, 1973.
} 
F. Flipo, Les inégalités écologiques et sociales: l'apport des théories de la justice, Mouvements, $n^{\circ} 60$, octobre-décembre 2009, pp. 59-76.

Claude Lefort ${ }^{82}$. Justice écologique, volonté d'inclure les écosystèmes dans une conception plus large de la justice ou "retour à la terre » vichyste voire nazi ${ }^{83}$ ? Dans ce cadre, le motif des « primitifs » ou des « autochtones » joue en Occident un rôle tout-à-fait particulier, bien différent de "l'état de nature » tant débattu au cours du siècle des Lumières : sont-ils une incarnation d'une sagesse oubliée de la 《 vie sauvage ${ }^{84}$ ou au contraire le symbole de la nostalgie du passé ? Les partis occidentaux voient plutôt la nature comme le lieu dont les êtres humains sont sortis grâce à la technique et à la croissance ${ }^{85}$. Les "Modernes", au sens étymologique de « ceux qui sont arrivés après », n'en finissent plus de mettre en doute leur identité et de chercher des normes pour guider leur action. Les débats sont donc extrêmement tendus, opposant la protection de "l'homme» à la protection de "la nature ». Du côté socialiste, la volonté de respecter la nature a généralement été comprise comme idéologique, mensongère, malthusienne : les partisans de la protection des écosystèmes, appartenant à la classe exploitante, chercheraient à préserver leurs rentes en faisant croire à la rareté des ressources. Toutefois aujourd'hui certains courants écosocialistes commencent à se référer à un «équilibre naturel ${ }^{86}$, tandis que d'autres reconnaissent la parenté inévitable de tout engagement écologiste sincère avec la « deep ecology $»^{87}$.

L'utilitarisme a connu les mêmes travers. Les physiocrates savaient que l'économie ne crée aucune richesse sur le plan écologique. J.S. Mill en 1848 estimait que le bien-être matériel dont jouissait son époque ne nécessitait guère d'amélioration, et que la vie serait désagréable s'il fallait trop travailler ${ }^{88}$. Mais le $19^{\text {ème }}$ siècle change cela et consacre la volonté de domination de la nature. J.-B. Say affirme en 1841 que la passion de domination de la nature est la plus «auguste» de toutes ${ }^{89}$. Ce n'est pas Adam Smith mais Say encore qui fait de la civilisation un résultat des progrès de la technologie ${ }^{90}$ et qui affirme que les ressources naturelles sont inépuisables ${ }^{91}$. Walras va en fournir une traduction mathématique, tout en se réclamant du socialisme ${ }^{92}$. A nouveau, la nature est le lieu de la conquête. Le donné se donne comme étant à transformer.

Les approches ancrées dans les théories des droits ne font guère mieux. L'hypothèse évite la question tant de l'international que de la « suffisance ». Rawls reconnaît ignorer l'écologie ${ }^{93}$. Il ignore largement les enjeux internationaux, sauf un opuscule tardif sur le droit des gens ${ }^{94}$. Rawls, lorsqu'il applique le voile d'ignorance aux représentants des sociétés, suppose que les

\footnotetext{
${ }^{82}$ Lefort C., L'invention démocratique, Paris, Fayard, 1994.

${ }^{83}$ Ferry L., Le nouvel ordre écologique, Paris, Grasset, 1992.

${ }^{84}$ Nash R., The Right of Nature - A History of environmental ethics, The University of Wisconsin

Press, 1967.

${ }^{85}$ Sainteny G., op. cit., p. 45.

${ }^{86}$ Wallis V., Vers le socialisme écologique, pp. 123-146, M. Löwy (coord.), Ecologie et socialisme, Paris, Syllepse, 2005, p. 132.

${ }^{87}$ Kovel J., Dialectique des écologies radicales, pp. 81-91, M. Löwy (coord.), Ecologie et socialisme, Paris, Syllepse, 2005, p. 82.

${ }^{88}$ Mill J.-S., Principes d'économie politique, 1848, Chapitre VI.

${ }^{89}$ Woo S.-H., Croissance économique et concept de limites dans la pensée économique, Thèse de l'Université Paris X-Nanterre, sous la direction de Michel Rosier, 1996, p.57.

${ }^{90}$ Ibid. p. 134

${ }^{91}$ Ibid. p. 130

${ }^{92}$ Albertini J.-M. \& A. Silem, Comprendre les théories économiques, Paris, Seuil, 2001, pp.124-125.

${ }^{93}$ Rawls J., Théorie de la justice, Paris, Gallimard, 1995b, Ed. orig. 1971, §77, p. 550.

${ }^{94}$ Rawls J., Le droit des gens, Paris, Editions Esprit, $1995 a$.
} 
F. Flipo, Les inégalités écologiques et sociales: l'apport des théories de la justice, Mouvements, $n^{\circ} 60$, octobre-décembre 2009, pp. 59-76.

sociétés sont auto-suffisantes et fermée ${ }^{95}$. La seule référence rawlsienne au long terme admet que la question est difficile et l'auteur n'y consacre que quelques pages ${ }^{96}$. A. Gosseries a essayé d'en tirer toutes les implications, il conclut qu'elle ouvre plus de problèmes qu'elle n'en résout ${ }^{97}$. Quand la nature apparaît dans les analyses rawlsiennes, c'est sous la forme des inégalités naturelles dont il estime qu'elles sont à compenser par les «biens sociaux premiers » dont W. Kymlicka montre qu'ils sont principalement conçus comme étant issus d'une coopération à avantages mutuels dans l'exploitation de la nature ${ }^{98}$.

L'autre point d'entrée, dans les théories des droits, est celui de l'appropriation initiale. Marx souligne la violence de l'expropriation initiale, des enclosures, à l'encontre des paysans, mais il en vient tout de suite à la question de la répartition des richesses supplémentaires générées par cet acte : il admet donc que cet acte a été plutôt bénéfique du point de vue agrégé. D'après W. Kymlicka, Locke et Nozick ont recours à la même explication ${ }^{99}$ : 1'appropriation est légitime si elle concourt à accroître l'ensemble des richesses disponibles. P. Manent reconnaît aussi que le droit d'appropriation peut aller au-delà de ce qu'on peut consommer car : «en convenant avec mes semblables d'un équivalent incorruptible des biens naturels corruptibles, par exemple l'or ou l'argent, alors l'accumulation pourra être sans limites puisqu'elle n'impliquera plus de gaspillage. Partant, en m'appropriant une portion de terre par mon travail, loin d'enlever au bien commun de l'humanité, je lui ajoute tous les fruits de la terre qui doivent leur existence à mon travail. [...] Et il est [dès lors] évident que personne d'autre n'a de droit sur ces biens qui ne sont pas donnés par la nature mais produits par mon travail $\gg{ }^{100}$. Ainsi l'or et l'argent seraient incorruptibles... et leur accumulation ajouteraient au bien commun de l'humanité ! De telles assertions sont, du point de vue écologique, dénuées de tout fondement. Au contraire l'écologie souligne que tout métal est voué à l'épuisement définitif, au contraire de la vie, des écosystèmes et des énergies renouvelables. L'or ne peut pas se manger, il n'entre pas dans la chaîne alimentaire, il ne joue aucun rôle clé dans les écosystèmes. Et l'appropriation d'une partie du cycle du carbone ou de l'eau enlève bel et bien une part du bien commun de l'humanité.

\section{L'écologie ignore le social}

Les théories de la «justice écologique » peuvent toutefois entrer en conflit avec les théories de la « justice sociale ».

Tout d'abord, l'écologie scientifique a longtemps exclu les activités humaines de son champ de recherches, même si l'on voit ici et là des tentatives pour mettre en place une « écologie humaine » au statut encore très ambigu. Ne fournissant guère de référentiel pour les zones habitées, les éthiques environnementales restent peu praticables dans les milieux dits «fortement artificialisés ». Le biocentrisme et l'écocentrisme ne semblent pas pouvoir accepter la présence des villes, qui sont pour elles un milieu désert, sans vie, et d'ailleurs certaines analyses écologistes ont explicitement dénoncé les villes comme anti-écologiques ${ }^{101}$.

\footnotetext{
${ }^{95}$ Rawls J., op. cit., 1995 b, p. 45

${ }^{96}$ Rawls J., op. cit., 1995b, §44

${ }^{97}$ Gosseries A., op. cit., p. 206-241.

${ }^{98}$ Kymlicka W., Les théories de la justice: Une introduction, Paris, La Découverte, 1999, p. 85.

${ }^{99}$ Kymlicka W., ibid., pp. 124-159.

${ }^{100}$ Manent P., Histoire intellectuelle du libéralisme, Paris, Calmann-Lévy, 1987, pp. 98-99.

${ }^{101}$ Younès C. (dir.), Ville contre-nature, Paris, La Découverte, 1999.
} 

Mouvements, $n^{\circ} 60$, octobre-décembre 2009, pp. 59-76.

Henry Thoreau s'en va vivre dans les bois ${ }^{102}$. Aldo Leopold base ses analyses sur ses expériences de chasseur ${ }^{103}$.

De plus les éthiques environnementales font souvent comme si tous les êtres humains étaient dotés des mêmes capacités et comme s'ils devaient rester attachés à l'écosystème dans lequel ils se trouvent à un moment donné. L'être humain est pris dans sa dimension générique de représentant d'une espèce, un « humain moyen » dans l'écosystème considéré, les inégalités entre humains et entre écosystèmes étant laissées de côté. Pourtant la plus grande partie du milieu dans lequel vivent les êtres humains a été construite par d'autres êtres humains, passés ou contemporains, et cela est d'autant plus vrai que la division du travail est poussée.

Enfin les éthiques environnementales s'intéressent peu aux liens de solidarité qui peut exister entre êtres humains et qui déterminent pour une grande part libertés dont chacun jouit. "L'homme moyen » convoqué par les éthiques environnementales est toujours adulte et en bonne santé, jamais en guerre avec ses voisins. Les zones "naturelles » dans lesquelles il évolue sont en libre-accès, il peut se déplacer à volonté. Les inégalités naturelles telles que le handicap sont peu abordées, alors qu'elles sont l'un des sujets centraux des théories « sociales » de la justice. Comme l'écologie s'appuie sur les concepts de chaîne alimentaire et sélection naturelle, les éthiques environnementales peuvent prêter le flanc à l'accusation de « darwinisme social ».

L'utilitarisme écologique de son côté traite généralement tous les consommateurs à partir de moyennes, comme s'ils avaient un pouvoir d'achat égal. A la manière des approches classiques de l'économie, elle appelle les consommateurs à préférer les produits biologiques, les produits éco-efficaces etc. bref ce qu'on appelle parfois la "consom'action » mais ne s'étend pas beaucoup sur d'éventuelles différences de prix. L'utilitarisme écologique focalise sur l'épuisement des ressources et la pollution mais pas toujours sur l'inégalité des personnes face à ces enjeux. Les pauvres, qui consomment déjà peu, ont quelques difficultés à entendre l'argument de limitation des besoins et de décroissance, et cela d'autant plus que l'une des seules formes d'inclusion sociale qui leur reste est justement la consommation. Un possesseur de $4 \times 4$ est-il aussi responsable face aux changements climatiques qu'un possesseur de Twingo ? La restriction de l'accès aux richesses naturelles rencontre l'argument marxiste est bien connu : les possédants utilisent l'argument de rareté pour limiter les possessions... des autres, jugeant que leur propre consommation est parfaitement justifiée, du fait de leur rang ou de leurs "intérêts de classe ». Même s'ils partagent le souci de décroître de manière collective, les pauvres craignent d'être les victimes désignées de la cure d'amaigrissement. Les théories écologiques de la justice se sont d'ailleurs construites très largement en dehors de toute référence marxiste ou marxienne ${ }^{104}$, qui reste un corpus important en matière de théories « sociales » de la justice.

L'approche selon les droits, privés ou collectifs, n'ouvre pas réellement la question de savoir qui sont les principaux bénéficiaires de la protection d'un patrimoine donné. Hans Jonas par exemple, le précurseur, en est resté à une approche de «l'homme» en général ${ }^{105}$, et ses propositions politiques, peu soucieuses du respect des libertés civiles et politiques, restent très controversées. C'est pourtant ici que l'approche sociale serait la plus aisée à conduire. Sur le plan empirique, il y a peu d'évaluation de la réglementation environnementale en termes d'inégalités sociales. Toutefois certaines inégalités sont flagrantes, en particulier dans l'espace international. La protection de la diversité biologique, par exemple, est plus facile à

\footnotetext{
102 Thoreau H.D., Walden ou la vie dans les bois, Paris, Gallimard, 1990, éd. Orig. 1854.

${ }^{103}$ Leopold A., Almanach d'un comté des sables, Paris, Aubier, 1995, Ed. orig. 1948.

${ }^{104}$ Chesnais F. \& C. Serfati, op. cit., 2004.

105 Jonas H., Le principe responsabilité, Paris, Flammarion, 1990, Ed. orig. 1979.
} 

Mouvements, $n^{\circ} 60$, octobre-décembre 2009, pp. 59-76.

réaliser en Afrique que dans nos pays. La sauvegarde des éléphants mobilise d'autant mieux les foules qu'aucun Français n'a à cohabiter avec les pachydermes. La situation devient plus tendue lorsqu'il s'agit d'ours dans les Pyrénées. L'urgence écologique est là, le propos n'est pas de le nier, bien au contraire, mais les efforts à faire ne semblent pas équitablement répartis. Les ours seraient peut-être mieux protégés si les citadins avaient moins de résidences secondaires venant «miter» les écosystèmes. Ce n'est pas le tiers de Francilien qui ne part jamais en vacances qui vient déranger les chamois à la période de reproduction...

Cette approche pose la question de la détermination des limites de la communauté, écologique et sociale. Tous les Terriens ne sont pas entièrement responsables de tous les écosystèmes, ni de toutes les richesses naturelles, de même que tous les Terriens ne sont pas engagés dans les mêmes réseaux de solidarité : la sécurité sociale française ne prend en charge qu'environ 60 millions de personnes, sur plus de 6 milliards et demi de Terriens. Les questions écologiques viennent brouiller les frontières des communautés. Qu'est-ce qui relève du bien commun de l'humanité ? Qu'est-ce qui relève de la souveraineté nationale ? Les Français pensent que l'éléphant doit être protégé, mais que penseraient-ils d'une délégation kenyane venant leur demander d'assurer la protection de l'ours? Ils répondraient probablement que la question de l'ours est française, tout comme les Brésiliens tendent à répondre que la question de l'Amazonie est de leur libre choix. Toutefois si la protection de la diversité biologique devenait une priorité, des liens de réciprocité devraient être établis : les Kenyans devraient pouvoir recevoir quelque chose en échange de leur coexistence avec les éléphants, par exemple la garantie que les Français coexisteront bien avec l'ours... un bien commun mondial pourrait ainsi émerger, sans exiger un Etat mondial.

La négociation sur les changements climatiques et bien d'autres le montrent : la question des «droits justes » sur les ressources, ce que Kant appelait la « possession intelligible ${ }^{106}$, est plus que jamais à l'ordre du jour. Et elle le sera d'autant plus que les biens essentiels gratuits, jadis accessibles même aux plus démunis, se feront rares. Le droit à un environnement sain est désormais inscrit dans la Constitution. A cet égard «l'espace environnemental» défini par Siebert en 1982 puis repris par Opschoor \& Van der Ploeg en $1990^{107}$, et décliné sous diverses formes (droits per capita sur le $\mathrm{CO} 2$ etc.) peut être une piste pour l'action. Elle rejoint certains aspects des analyses de Marx, les théoriciens de la dépendance (R. Prebisch) ou les théoriciens de l'échange inégal (S. Amin), qui s'intéressent plus à la répartition des richesses qu'à leur niveau global. C'est souvent le point de vue de théoriciens du «Sud » tels que Tariq Banuri $^{108}$. L'enjeu ici est de protéger l'environnement au niveau local, parce que les populations n'ont pas les moyens ni de migrer ni de se payer les technologies de dépollution ou de restauration de l'écosystème si celui-ci vient à s'effondrer. Elles ont donc un besoin vital des services gratuits de la nature.

\section{Inégalités écologiques et inégalités sociales : vers une intégration ?}

La classification des géographes, qui opposent désormais les espaces « naturels » aux espaces «artificialisés », est significative de nouvelles polarités qui émergent au sein de l'interprétation publique du monde. Ces nouvelles polarités donnent lieu à la discussion de nouvelles inégalités au sein des sociétés : l'accès aux espaces verts remplace le droit à quitter le monde rural etc. En découlent des discussions sur la légitimité de ces inégalités, ainsi W. Pelletier qui montre que l'accès aux espaces verts bénéfice d'abord aux classes aisées ${ }^{109}$.

\footnotetext{
${ }^{106}$ Kant, Doctrine universelle du droit, Paris, Vrin, 1797, §1.

${ }^{107}$ Hille J., The concept of environmental space, European Environment Agency, 1997/2, p. 7.

${ }^{108}$ Banuri T., The South and the governability of the planet : a view from the South, pp. 405-412, Theys

J. (dir.), L'environnement au 21ème siècle - enjeux, Paris, Germes, 1998.
} 
F. Flipo, Les inégalités écologiques et sociales: l'apport des théories de la justice, Mouvements, $n^{\circ} 60$, octobre-décembre 2009, pp. 59-76.

Articuler inégalités sociales et inégalités écologiques est difficile. Cela tient à trois éléments, que nous proposons à la discussion en conclusion. Le premier tient à l'évolution de la définition du bien commun. Le déplacement de l'attention collective des inégalités sociales vers les inégalités écologiques révèle en creux un déplacement des sources de richesse capables de contribuer au bien commun. Les discussions autour des indicateurs de richesse confirment cela, en particulier le succès récent de l'empreinte écologique ou de la comptabilité « $\mathrm{CO} 2$ ». La nature, de source de maux, devient source de biens. Le second élément est la transnationalisation grandissante des enjeux. Le caractère très parcellaire de l'information, des contextes et des références communes ne facilite guère l'établissement de conventions partagées. Le troisième est le caractère historiquement situé des catégories de « social » et « écologie », qui signalent des segments du monde difficilement compréhensibles dans d'autres représentations. La difficulté à articuler le "social» et "l'écologique » donne d'ailleurs naissance à de nouveaux mouvements, tels le «sociétalisme » (http://www.societal.org ).

${ }^{109}$ Pelletier W., Sociale « nature » - Représentations de l'écologie et usages sociaux des environnements, pp. 179-190, Harribey J.-M. \& M. Loewy (dir.), Capital contre nature, Paris, PUF, p. 188, 2003. 
F. Flipo, Les inégalités écologiques et sociales: l'apport des théories de la justice, Mouvements, $n^{\circ} 60$, octobre-décembre 2009, pp. 59-76.

Albertini J.-M. \& A. Silem, 2001, Comprendre les théories économiques, Paris, Seuil.

Arendt A., 1973, Sur l'antisémitisme, Paris, Calmann-Lévy.

Audard C., 1996, Justice, pp. 781-789, M. Canto-Sperber (dir.), Dictionnaire d'Ethique et de Philosophie Morale, Paris, PUF.

Audard C., 1996, Utilitarisme, pp. 1563-1570, M. Canto-Sperber (dir.), Dictionnaire d'Ethique et de Philosophie Morale, Paris, PUF.

Ayres R.U. \& L.W., 1999, Accounting for resources 2 - The life cycle of materials, Edward Elgar Publishing.

Banuri T., 1998, The South and the governability of the planet : a view from the South, pp. 405-412, Theys J. (dir.), L'environnement au 21ème siècle - enjeux, Paris, Germes.

Bateson G., 1995, Vers une écologie de l'esprit, Paris, Seuil.

Beck U., 2003, La société du risque - sur la voie d'une autre modernité, Paris, Flammarion.

Belpomme D., 2004, Ces maladies créées par l'homme, Paris, Albin Michel.

Bentham J., 1780, Introduction to the Principles of Morals and Legislation, chap. 17.

Berten A., 1996, Déontologisme, pp. 377-383, M. Canto-Sperber (dir.), Dictionnaire d'Ethique et de Philosophie Morale, Paris, PUF.

Birnbacher D., 1998, La responsabilité envers les générations futures, Paris, PUF.

Bookchin M., 1992, Une société à refaire, Lyon, Atelier de création libertaire.

Bringzu S. \& H. Schütz, 2001, Total material requirements of the European Union, Technical Report no 55, European Environment Agency, 2001.

Cabeza-Gutés M. \& J. Martinez-Alier, 2001, L'échange écologiquement inégal, M. Damian \& J.-C. Graz, Commerce international et développement soutenable, Paris, Economica.

Carson R., 1962, Silent Spring, Boston, Houghton Mifflin.

Chesnais F. \& C. Serfati, 2004, L'origine et la nature des menaces pesant sur la reproduction de la vie de classes et de pays donnés: quelques outils analytiques et fils conducteurs marxistes. http://www.europe-solidaire.org/spip.php?article891.

Cochet Y., 2006, Pétrole apocalypse, Paris, Fayard.

Costanza R. \& al., 1997, The value of the world's ecosystem services and natural capital, Nature, vol. 387, pp. 253-260.

Daly H., 1996, Beyond Growth, Boston, Beacon Press.

Daly H.E. \& J. Cobb Jr., 1989, For the Common Good, Boston, Beacon Press.

Dauzat A., 1938, Dictionnaire étymologique de la Langue Française, Paris, Larousse.

Deléage J.-P. , 1992, Histoire de l'écologie, Paris, La Découverte.

Dobson A., 2003, Social justice and environmental sustainability, pp. 83-95, Agyeman J., Bullard R. D.

\& B. Evans, Just sustainabilities - Development in an unequal world, London, Earthscan, p. 85.

Dunlap R. E., 2002, The coming age of environmental sociology : an american perspective, pp. 25-40, Europaea, 1-2, anno VIII.

Faber D.R. \& D.McCarthy, 2003,Neo-liberalism, globalization and the struggle for ecological democracy: linking sustainability and environmental justice, pp. 37-63, Agyeman J., Bullard R.

D. \& B. Evans, Just sustainabilities - Development in an unequal world, London, Earthscan.

Ferry L., 1992, Le nouvel ordre écologique, Paris, Grasset.

Flipo F., 2004, Les tensions constitutives du « développement durable, Développement Durable et Territoires, avril.

Flipo F., 2004, La question de l'équité dans le cas du changement climatique, Ethique et économique/Ethics and Economics, 2 (1), http://Ethique-economique.org

Fritsch P., 1998, Effets croisés de réflexivité : prises de position problématiques sur la sauvegarde de la faune sauvage, pp. 81-93, Aubert F. \& J.-P. Sylvestre (dir.), Ecologie et société, Paris, Educagri Editions.

Georgescu-Roegen N., 1979, La décroissance, Paris, Editions Sang de la Terre.

Godard O., 1994, Le concept d'environnement, une hiérarchie enchevêtrée, pp. 97-112, Larrère C. \& R., La crise environnementale, Paris, INRA Editions.

Gosseries A., 2004, De l'affaire Perruche a la réforme des retraites, Paris, Aubier-Flammarion. 
F. Flipo, Les inégalités écologiques et sociales: l'apport des théories de la justice, Mouvements, $n^{\circ} 60$, octobre-décembre 2009, pp. 59-76.

Guha R. \& J. Martinez-Alier, 2000, Varieties of environmentalism, London, Earthscan, Ed. Orig. 1997. Hardin G.W., 1968, The Tragedy of the Commons, Science, n¹62, pp. 1243-1248.

Harribey J.-M., 2001, Marxisme écologique ou écologie politique marxienne, pp. 183-200, Bidet J., Kouvélakis E. (dir.), Dictionnaire Marx contemporain, Paris, PUF, Actuel Marx Confrontation.

Haut Conseil à l'Intégration, 1998, Lutte contre les discriminations: faire respecter le principe d'égalité. Rapport au Premier Ministre, Paris, La Documentation Française.

Hille J., 1997, The concept of environmental space, European Environment Agency, 1997/2, p. 7.

Hornborg A., 2003, Cornucopia or zero-sum game? The epistemology of sustainability, Journal of World-Systems, vol. 9, n², pp. 205-216.

Hornborg A., 2006, Footprints in the cotton fields: The Industrial Revolution as time-space appropriation and environmental load displacement, Ecological Economics, n59, pp. 74-81.

IFEN, 2001, Signaux précoces et leçons tardives: le principe de précaution 1896-2000, Agence Européenne de l'Environnement.

Iselin F., 2005, Ecologie et conscience de classe, pp. 33-43, M. Löwy (dir.), Ecologie et socialisme, Paris, Syllepse.

Jancovici J.-M., 2005, Combien suis-je un esclavagiste ? http://www.manicore.com/documentation/esclaves.html

Jonas H., 1990, Le principe responsabilité, Paris, Flammarion, Ed. orig. 1979.

Kant, 1797, Doctrine universelle du droit, Paris, Vrin.

Kaul I., Grunberg I. \& A. Stern, 1999, Global Public Goods - International Cooperation in the $21^{\text {st }}$ Century, Oxford, Oxford University Press.

Kovel J., 2005, Dialectique des écologies radicales, pp. 81-91, M. Löwy (coord.), Ecologie et socialisme, Paris, Syllepse.

Kymlicka W., 1999, Les théories de la justice: Une introduction, Paris, La Découverte.

Larrère C. \& R., 1997b, Du bon usage de la nature, Paris, Aubier, 1997.

Larrère C., 1997a, Les philosophies de l'environnement, Paris, PUF, 1997.

Larrère C. \& L., 1999, Environnement, pp. 360-363, in D. Lecourt (dir.), Dictionnaire d'histoire et de philosophie des sciences, Paris, PUF.

Latouche S., 2003, Justice sans limites - le défi de l'éthique dans une économie mondialisée, Paris, Fayard.

Lefort C., 1994, L'invention démocratique, Paris, Fayard.

Leopold A., 1995, Almanach d'un comté des sables, Paris, Aubier, Ed. orig. 1948.

Manent P., 1987, Histoire intellectuelle du libéralisme, Paris, Calmann-Lévy.

Marten G.G., 2001, Human ecology - basic concept for sustainable development, London, Earthscan.

Martinez-Alier J., 1998, Ecological Debt - External Debt, http://www.cosmovisiones.com/DeudaEcologica/a_alier01in.html

Martinez-Alier J., 2002, The environmentalism of the poor, A report for UNRISD for the WSSD Summit,. http://www.foe-scotland.org.uk/nation/ej_alier.pdf

Marx K., 1993, Le Capital, Paris, PUF, Ed. orig. 1872.

Mill J.-S., 1848, Principes d'économie politique.

Miller D., Coleman J., Connolly W. \& A. Ryan (ed.), 1989, Social-démocratie, pp. 713-719, Dictionnaire de la pensée politique - Hommes et idées, Paris, Hatier, Ed. orig. London, Basil Blackwell, 1987.

Muradian R. \& J. Martinez-Alier, 2001, Trade and the environment : from a « Southern » perspective, Ecological Economics, n³6, pp. 281-297.

Nash R., 1967, The Right of Nature - A History of environmental ethics, The University of Wisconsin Press.

O'Connor J., 2003, La seconde contradiction du capitalisme : causes et conséquences, pp. 57-66, Harribey J.-M. \& M. Loewy (dir.), Capital contre nature, Paris, PUF.

Ost F., 1995, La nature hors la loi. L'écologie à l'épreuve du droit, Paris, La Découverte.

Paredis E. \& al., 2004, Elaboration of the concept of ecological debt, VLIR-BVO project 2003, Final report. http://cdonet.rug.ac.be/noordzuid/onderzoek/ecological_debt/ecodebt_report_1_core.pdf 
F. Flipo, Les inégalités écologiques et sociales: l'apport des théories de la justice, Mouvements, $n^{\circ} 60$, octobre-décembre 2009, pp. 59-76.

Passet R., 1979, L'économique et le vivant, Paris, Economica.

Pelletier W., 2003, Sociale "nature » - Représentations de l'écologie et usages sociaux des environnements, pp. 179-190, Harribey J.-M. \& M. Loewy (dir.), Capital contre nature, Paris, PUF.

Pettit P., 1996, Conséquentialisme, pp. 313-320, M. Canto-Sperber (dir.), Dictionnaire d'Ethique et de Philosophie Morale, Paris, PUF.

Polanyi K., 1983, La grande transformation - Aux origines politiques et économiques de notre temps, Paris, Gallimard, Ed. orig. 1944.

Ramade F., 2003, Eléments d'écologie - 2 tomes, Paris, Ediscience international.

Rawls J., 1995a, Le droit des gens, Paris, Editions Esprit.

Rawls J., 1995b, Théorie de la justice, Paris, Gallimard, Ed. orig. 1971.

Sainteny G., 2000, L'introuvable ecologisme français, Paris, PUF.

Sen A., 1992, Inequalities re-examined, Oxford, Clarendon Press.

Sen A., 2004, L'économie est une science morale, Paris, La Découverte.

Singer P., 1993, La libération animale, Paris, Grasset.

Solow R. M., 1974, The Economics of Resources or the Resources of Economics, pp. 1-14, The American Economic Review, vol. 64, n², Nashville, May, p. 10.

Taylor Paul W., 1983, In Defense of Biocentrism, Environmental Ethics, Vol. 5, No. 3 (Automne), pp. 237-243.

Theys J., 2005, Les inégalités écologiques, dimension oubliée de l'action publique : entre raisons politiques et explications épistémologiques, Colloque «Inégalités sociales et environnementales », Institut d'Urbanisme de Paris.

Thoreau H.D., 1990, Walden ou la vie dans les bois, Paris, Gallimard, éd. Orig. 1854.

Toth F. L. (Ed.), 1999, Fair Weather? Equity concerns in Climate Change, Earthscan, London.

Vadrot P.-M. , 1973, Déclaration des droits de la nature, Paris, Stock.

Vivien F.-D., 2005, Le développement soutenable, Paris, La Découverte.

Wallis V., 2005, Vers le socialisme écologique, pp. 123-146, M. Löwy (coord.), Ecologie et socialisme, Paris, Syllepse.

William J. Clinton, 1994, Executive order 12898, February 11.

WWF, 2006, Living Planet Report.

Wackernagel M. \& W. Rees, 1996, Notre empreinte écologique, Montréal, Ecosociété

Woo S.-H., 1996, Croissance économique et concept de limites dans la pensée économique, Thèse de l'Université Paris X-Nanterre, sous la direction de Michel Rosier.

Younès C. (dir.), 1999, Ville contre-nature, Paris, La Découverte.

Zarsky L. (Ed.), 2002, Human rights and the environment - conflicts and norms in a globalizing world, London, Earthscan.

Zuindeau B., 2005, La «loi de Kuznets»: de l'économie de la répartition à l'économie de l'environnement, Colloque international Association Charles Gide pour l'Etude de la Pensée Economique. 